\title{
EACO: AN ENHANCED ANT COLONY OPTIMIZATION ALGORITHM FOR TASK SCHEDULING IN CLOUD COMPUTING
}

\author{
Surabhi Sharma ${ }^{1 *}$ and Richa Jain ${ }^{2}$ \\ ${ }^{1,2}$ Banasthali Vidypith, Tonk, Rajasthan, India \\ ${ }^{1}$ surabhitanu96@gmail.com, ${ }^{2}$ jainricha86@gmail.com
}

\begin{abstract}
Cloud Computing is emerging as an influential architecture to perform complex and large scale computing. It provides on-demand access to services on the "Pay-as-you-go" method. In cloud computing environment Task Scheduling is an essential technique that is required for allocating tasks to appropriate resources for proper resource utilization and optimizing overall system performance. Task scheduling is an NP-hard problem. Many researchers have proposed various algorithms like ACO, PSO, GA, and Bat etc to get optimal results. In this paper, the enhanced Ant Colony Optimization (EACO) algorithm has been proposed that serves improved task scheduling with minimum makespan while maintaining cost. This algorithm mainly contributes in minimizing total completion time for scheduling tasks on resources. This is attained by splitting the ordered submitted tasks into bunches -the sub list of tasks. The main goal of EACO is to minimize total execution time. The proposed algorithm EACO is simulated using the CloudSim toolkit and compared with the existing nature-inspired algorithm. The experimental results show that the presented algorithm improves results in terms of makespan.
\end{abstract}

Keywords - cloud computing, task scheduling, ant colony optimization (ACO), makespan

\author{
Abbreviation: \\ ACO Ant Colony Optimization \\ VM Virtual machine \\ uc Utilization condition \\ ET Execution time
}

\section{INTRODUCTION}

Cloud computing is growing as an emerging technology, booming in the area of distributed computing. It refers to services and applications that run on a distributed network accessed by using common internet protocols and standards [2]. Cloud Computing provides on-demand access to the pool of configurable resources that can be anything from software to infrastructure on the basis of the pay-per-use model with minimum interaction between the cloud service provider and end-users over the internet. It delivers services to users using SLA (Service-Level- Agreement) by satisfying QoS (Quality-of-Service) requirements. Resources are easily provisioned and released with minimum management. It has become an influential model for performing large scale 
complex computing and service provisioning in a distributed environment without possessing and managing the complexity of underlined technology.

Task scheduling is the most critical technologies in a cloud computing environment. It is a scheduling policy for tasks where-

- $\quad$ Task is the work that should be done in a particular time period and

- $\quad$ Scheduling is the process of allocating appropriate resources to particular jobs and job is divided into multiple tasks.

Task scheduling in cloud computing is emerging as a challenge for getting minimum execution time and maximum resource utilization while maintaining QoS [12]. Task scheduling in the cloud environment is an NP-hard problem and many researchers have developed methods to solve this problem.

In the proposed work enhanced ant colony optimization algorithm is evolved that improves basic ACO and put forth for achieving reduced makespan by scheduling a large number of ordered tasks in the form of bunches of tasks. The proposed algorithm sorts submitted tasks on the basis of their length and split them into a number of bunches that is a sub-list of submitted tasks and finally schedules tasks in bunches.

The rest of the paper is composed as section II presents the literature review. Section III presents ACO algorithm and proposed EACO algorithm. Section IV describes simulation results and comparison with primary ACO. Finally, Section V presents the conclusion and future work.

\section{RELATED WORKS}

T Mathew et al. [4] presented a detailed study of various task scheduling algorithms and related parameters. They classify these scheduling methods into three groups workflow scheduling, task scheduling and resource scheduling but the focus is only set on task scheduling methods. Task scheduling methods illuminated and categorized as Heuristic approach, Energy-efficient approach, Hybrid approach with different parameters used for scheduling algorithms and their benefits and drawbacks are also provided for better comprehension of these methods. This is fruitful for developing a new scheduling algorithm in the cloud environment.

Dorigo, Marco, and Gianni Di Caro [5] proposed an algorithm "Ant colony optimization" based on the behavior of ant colony. They presented a basic description of ant colony optimization metaheuristic techniques and give applications of proposed work.

James Kennedy and Russell Eberhart [6] proposed an algorithm "Particle Swarm Optimization" based on simulation of the social model. PSO is based on two methodologies- artificial life and bird flocking/fish swarming theory. The motivation for simulation is human social behavior because human adjusts physical, cognitive and experience variables. This algorithm is used for the optimization of non-linear functions and neural network training. It has a relation with GA and artificial life and lies between evolutionary programming and GA. PSO is easy to develop as it requires a few lines of code than GA. It requires primitive mathematical operators, less computation in terms of less storage and power and performs efficient task scheduling in the cloud environment.

$\mathrm{Li}$, Kun, et.al [7] presented an improved ant colony optimization algorithm for load balance in task scheduling. LBACO is proposed for a balancing load on resources in the cloud environment during task scheduling. It also reduces makespan and maintains cost. It is compared with other algorithms and outperforms better also in the case of different sizes of tasks.

Tawfeek, Medhat A., et.al [8] presented a comparative study of ant colony optimization for task scheduling in cloud computing. A comparison on the basis of 
makespan is done. The presented study results that ACO results with minimum makespan in comparison with FCFS and RR.

Moon, YoungJu, et.al [9] proposed improved slave ants based ACO. It works on the strategy diversification and reinforcement of slave ants. It improves results and remove global optimization problem using slave ants that prevent by avoiding tour on long paths that are wrongly accumulated by leading ants. It results better than ACO, IACO and serve better resource utilization but lacking cost.

Jain R et.al [10] discussed a great and methodical study of nature-inspired algorithms for workflow scheduling in the heterogeneous cloud computing environment. It also provides the classification of nature-inspired algorithms as ACO, PSO, GA, ABC, BAT algorithms but major work is done with PSO and GA. It is mainly considering cost and time as parameter for analyzing these algorithms but security, energy consumption, load balancing is ignored. It illuminates objectives, characteristics, and limitations of these methods, and offers a complete understanding of nature-inspired algorithms with awareness of unsolved issues related to workflow scheduling in cloud environment and future trends in the field. It also offers a brief study of enhanced versions of these algorithms proposed by other scholars.

Gamal Abd El-Nasser A. Said [11] presented brief study to solve resource allocation problem in cloud computing environment to get high performance and efficient utilization. This paper presents a survey of various metaheuristic methods on the basis of ACO, PSO, and GA to solve scheduling problems by reducing makespan, execution cost, load balancing and proper resource utilization.

Saleh H et.al [12] proposed improved PSO (IPSO) for scheduling large no. of task with proper resource utilization and load balance. It works by making batches on the basis of length and total no. of provided tasks and get best optimal solution from these batches to further process to get best optimal solution form given sub-optimal solutions. It balances load and provides proper resource utilization. IPSO is compared with PSO, ACO, honey bee and round robin. It results that IPSO performance better than these methods in terms of reducing makespan, degree of imbalance of tasks and VMs, and standard deviation of load.

Reddy G. Narendrababu et.al [1] presented a modified ant colony optimization algorithm MACO for multi-objective task scheduling in cloud environment. MACO is an improved ACO and works on assigning pheromone

value to VMs that has great processing speed, bandwidth, RAM, storage, makespan etc. for proper task allocation to efficient VMs. It assigns task efficiently to available VMs for better resource utilization and to reduce degree of imbalance. It outcomes better makespan, good entire system load balance, performance and efficiency in assigning tasks to VMs in comparison with basic ACO,PSO and GA.

AA Nasr et.al [14] proposed CR-AC hybrid algorithm for workflow scheduling in cloud environment for low cost and minimized scheduling time under defined constraints. It processes in phases where CRO offers near optimal solution and ACO take it as input and improves quality of given optimal solution. CR-AC performs better than $\mathrm{CRO}, \mathrm{ACO}$, modified PSO and CEGA algorithms and serves reduced cost, scheduling time and meeting deadline constraints with improved efficiency and performance.

M Kalra and S Singh [15] reviewed applications of existing metaheuristic scheduling techniques - Ant Colony scheduling algorithm, Particle Swarm scheduling algorithm, Genetic algorithm, and two novel techniques- League Championship algorithm and BAT algorithm, for scheduling resources in cloud environment. They also presented views on the work done by others in cloud for solving resource scheduling problem using these metaheuristic methods. Based on the survey, they found some observations and open issues related to these methods. These methods provide improved convergence speed, quality of solution and enhanced efficiency \& performance of system. This research is 
beneficial for understanding nature and related issues of resource allocation, task scheduling, load- balancing and other problems in cloud environment.

\section{ANT COLONY OPTIMIZATION ALGORITHM}

$\mathrm{ACO}$ is a population-based metaheuristic technique used for finding optimal solutions for difficult optimization problems. ACO works for simulation by following the basic idea of foraging behavior of ant colonies. Marco Dorigo and colleagues introduced the first ACO algorithms in the early 1990's. Ants are social insects and they live in colonies where they focus on the goal of colony survival rather than individual survival. For survival ants find food with shortest path from the nest. In this method of finding food with shortest path, ants use a chemical known as pheromone. Ants roam in the search of food and randomly explore the area, when ants move from nest to food location they leaves pheromone on the way. As they get food with minimum distance from nest, they return from the trip. When a food source with minimum distance is found ants may travel fast as compare to other food sources. Pheromone will be highly consisted on the shortest path and ants can smell pheromone. In this way ants find out an optimal solution for finding food for colony. In cloud computing ACO is used to minimize execution time for scheduling large number of tasks.

\section{THE PROPOSED EACO TASK SCHEDULING ALGORITHM}

EACO is an improved ACO that performs to optimally schedule tasks on available resources with minimum total completion time while maintaining cost with quality of service. In cloud computing environment datacenters works as resource provider and virtual machines are actual resources. Task scheduling in cloud computing is the process of scheduling submitted tasks properly on available VMs. The main aim of proposed EACO is to minimize makespan and maximize resource utilization while maintaining cost.

In proposed algorithm a number of datacenters are considered and each datacenters hold a number of hosts. Each host contains a number of virtual machines that is a resource in cloud computing. A host can contains m number of VMs and each VM consists of mips, ram, vmm, pesNumber etc. The proposed algorithm represents ith resource with VMi as follows:

$$
\mathrm{VM}=\mathrm{VMi} \quad \text { where } \mathrm{i}=1,2,3, \ldots . . \mathrm{n} \text {; }
$$

The proposed algorithm considers $\mathrm{m}$ number of independent tasks that contains different sizes. EACO represents these tasks with taskj that consists length, pesNumber, file size etc. that is represented as follows:

$$
\text { Task }=\text { taskj } \quad \text { where } \mathrm{j}=1,2,3, \ldots \ldots . \mathrm{m} \text {; }
$$

Scheduling in cloud is the process of allocating these m number of tasks on available $n$ number of VMs. Scheduler performs scheduling process and creates an allocation plan that presents which task is allocated to which VM. The allocation matrix is represented as follows:

$$
a p=\left[\begin{array}{cccc}
a p_{1,1} & a p_{1,2} & \ldots & a p_{1, m} \\
a p_{2,1} & a p_{2,2} & \ldots & a p_{2, m} \\
\ldots & \ldots & \ldots & \ldots \\
a p_{n, 1} & C_{n, j} & \ldots & C_{n, m}
\end{array}\right]
$$


Allocation matrix shows that which task is scheduled on which VM and assumes that only one task is assigned to only one VM. If a task is allocated to a VM than a binary variable is set to 1 otherwise set to 0 . That is shown below:

$$
a p_{i}=1 \text { if }\left\{\begin{array}{l}
v m_{i} \text { assigned to task } k_{j} \\
0 \quad \text { otherwise }
\end{array}\right.
$$

Now for scheduling tasks using EACO, first tasks arrives, and a sorting method is applied on the submitted task to get ordered task list on the basis of the length of tasks. In sorting method lengths of tasks available in task list is compared and ordered in ascending order.

At the second step this ordered task list is split into bunches and passed for allocation method that allocates these tasks to appropriate resources. Before creating bunches to get maximum utilization, it is checked that bunch length must be less than total resource capacity i.e., resource utilization state indicator. It can be calculated as follows:

$$
\mathrm{UC}=\sum_{i=1}^{n} V M_{i}(\mathrm{MIPS}) / \mathrm{n}
$$

So, based on the available resource's tasks are scheduled for better resource utilization and reduced makespan. Tasks are divided into bunches and each bunch length is determined by the following formula:

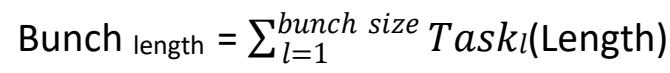

Bunches are created based on available resources and task collection in bunches is terminated when bunch length violates the total mean of available resources that is checked by :

$$
\text { Bunch length } \leq \mathrm{uc}
$$

Now, after creating bunches with all the conditions, these bunches are passed to the allocation task method that allocates tasks to proper virtual machines by applying ACO. For task scheduling in cloud computing, all ants are assigned with starting VMs randomly. Then they moved from one VM to other for optimal solution until all tasks are allocated to VMs. Iteration is assigned with index 1 to tmax i.e. maximum number of tasks. ACO performs basic operations that are initialize pheromone, choose VM for the next task and update pheromone.

Our main goal in the proposed algorithm is to minimize total makespan or total execution time for scheduling tasks while maintaining cost and maximize resource utilization. ACO calculates execution time for every task. Execution time for each task is calculated as follows:

$$
\begin{gathered}
E T=\left(\text { Task }_{j}(\text { Length }) / V_{i}(\text { NumberofPes }) * V M_{i}(\text { MIPS })\right)+\left(\text { Task }_{j}\right. \\
\left.(\text { Filesize }) / V M_{i}(B w)\right)
\end{gathered}
$$$$
\text { Where } \quad \mathrm{i}=1,2,3, \ldots \ldots, \mathrm{n} \text { and }
$$$$
\mathrm{j}=1,2,3, \ldots \ldots . . \mathrm{m} \text {. }
$$

ACO is initialized with ordered sub-list and pheromone is initialized with these tasks and VMs. These tasks are shuffled on VMs and an optimal solution is found out. Every time when a better solution is found, pheromone is updated that means that every time scheduling plan is checked and if it gives better execution time then scheduling strategy is 
updated with this new one allocation plan. EACO repeats these steps every time and gets best optimal solution. Algorithm for EACO is given below:

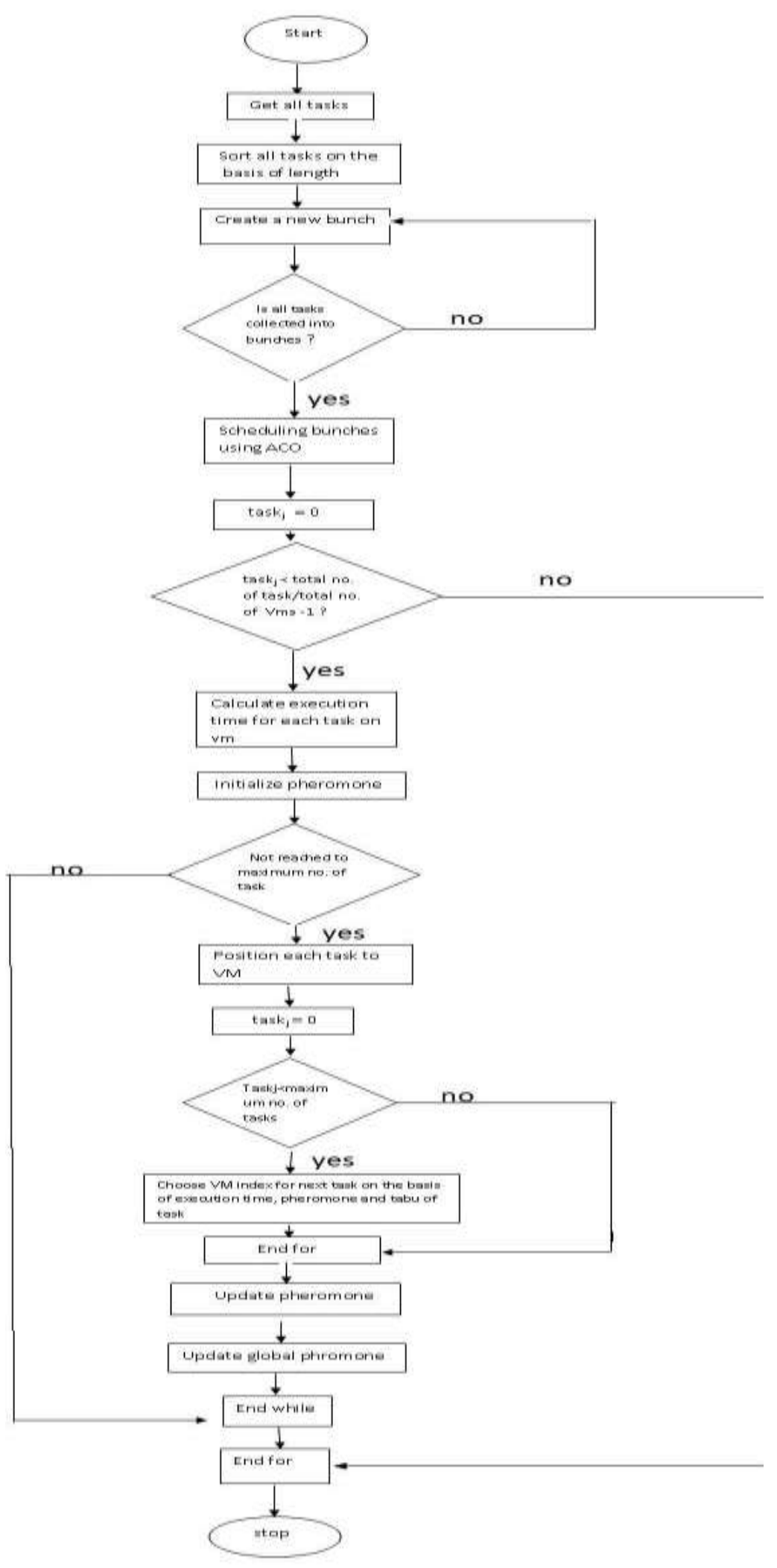

Fig. 1 Flow Chart of EACO 


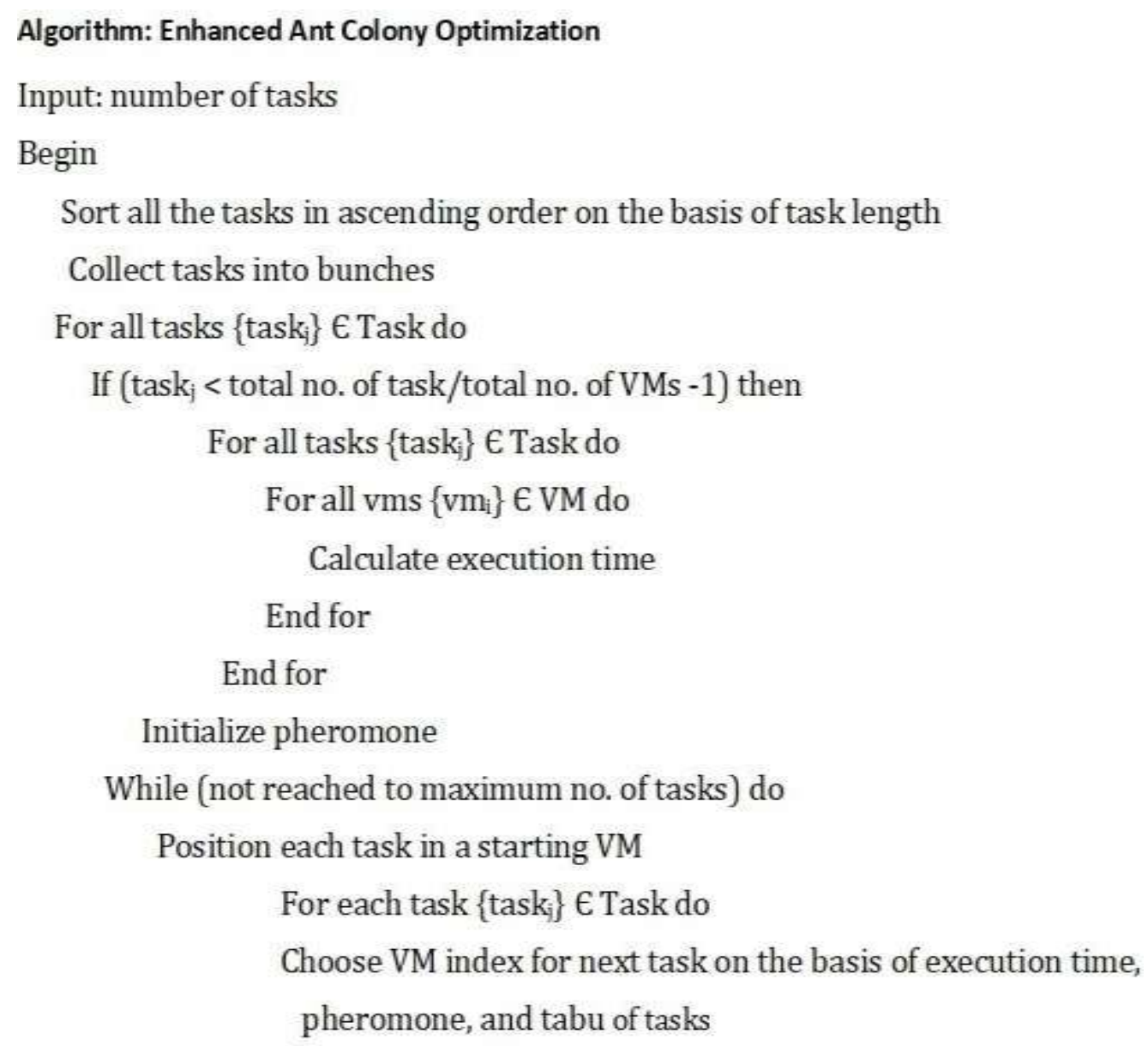

End for

Update the pheromone

Update global pheromone

End while

End if

End for

End

Output: reduced makespan

\section{EXPERIMENTS AND RESULTS}

To evaluate EACO CloudSim 3.0.3 toolkit is used that model and simulates large number of tasks in cloud computing. On the basis of simulation results, the performance and efficiency of the proposed algorithm is measured.

\subsection{EXPERIMENTAL SETUP}

CloudSim is a simulation and modeling toolkit that is used to evaluate the performance of the proposed algorithm and compare with other algorithms in the terms of makespan. Simulation environment is shown below: 
Table I. Experiment Environment

\begin{tabular}{|c|c|c|}
\hline Type & Parameters & Value \\
\hline VM & $\begin{array}{l}\text { Processor speed } \\
\text { Memory } \\
\text { Bandwidth } \\
\text { Image size } \\
\text { PesNumber } \\
\text { VMM }\end{array}$ & $\begin{array}{l}1000 \\
512 \mathrm{MB} \\
1000 \\
10000 \\
1 \\
\text { Xen } \\
\end{array}$ \\
\hline Host & $\begin{array}{l}\text { MIPS } \\
\text { Storage } \\
\text { VMM Monitor } \\
\text { Memory } \\
\text { Bandwidth }\end{array}$ & $\begin{array}{l}1000 \\
1000000 \\
\text { Xen } \\
25600 \mathrm{MB} \\
50000\end{array}$ \\
\hline $\begin{array}{l}\text { Data } \\
\text { Center }\end{array}$ & $\begin{array}{l}\text { Arch } \\
\text { Operating system } \\
\text { VMM } \\
\text { Time_zone } \\
\text { Cost } \\
\text { Cost Per Memory } \\
\text { Cost Per Storage } \\
\text { Cost Per Bandwidth }\end{array}$ & $\begin{array}{l}\text { X86 } \\
\text { Linux } \\
\text { Xen } \\
10.0 \\
3.0 \\
0.05 \\
0.1 \\
0.1\end{array}$ \\
\hline $\mathrm{ACO}$ & Population size & 100 \\
\hline
\end{tabular}

\subsection{EXPERIMENT RESULTS}

To evaluate performance of proposed algorithm, we compared EACO with other existing metaheuristic techniques ACO and PSO. CloudSim results that EACO performs better than ACO in terms of makespan. The experiment used varying number of tasks and VMs accordingly.

The experiment uses a varying number of tasks from 1000 to 20000.

Table II. Comparison of proposed EACO algorithm with ACO algorithm

\begin{tabular}{llll}
\hline $\begin{array}{l}\text { Number of } \\
\text { Cloudlets }\end{array}$ & $\begin{array}{l}\text { Number } \\
\text { of VMs }\end{array}$ & Makespan & \\
\hline & & ACO & EACO \\
\hline $\mathbf{1 0 0 0}$ & 10 & 99.92 & 93.88 \\
\hline $\mathbf{5 0 0 0}$ & 25 & 198.32 & 194.56 \\
\hline $\mathbf{1 0 0 0 0}$ & 50 & 221.21 & 216.21 \\
$\mathbf{2 0 0 0 0}$ & 100 & 224.46 & 221.35 \\
\hline
\end{tabular}


Above table shows the comparison results of ACO and EACO. It shows EACO results better makespan than basic ACO.

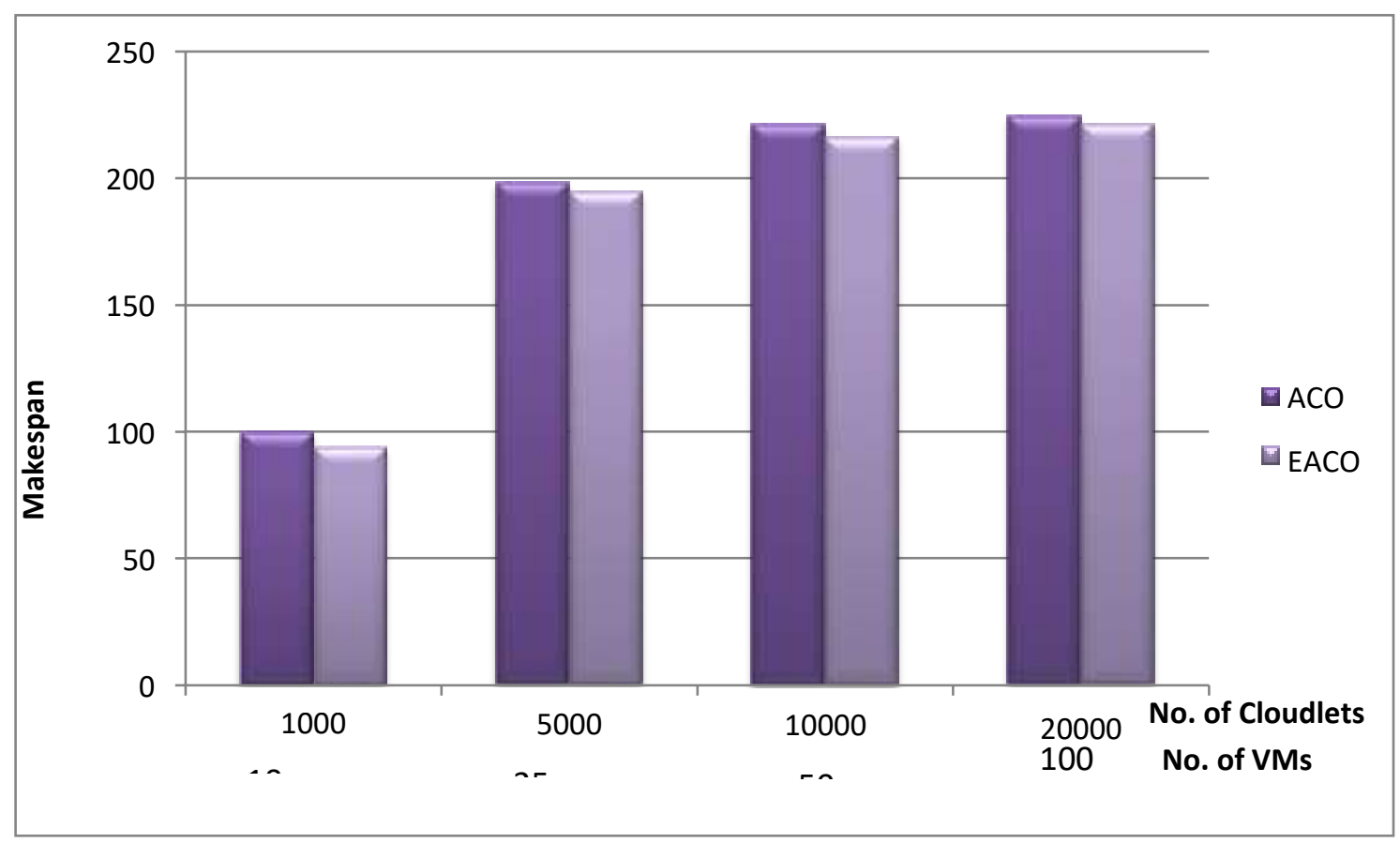

Fig. 2 Makespan time versus number of Cloudlets

\section{CONCLUSION AND FUTURE WORK}

To perform efficient task scheduling with minimized completion time and proper resource utilization a effective scheduling algorithm is required. In this paper a improved ant colony optimization has been developed that results better than basic ACO in terms of total completion time with resource utilization. EACO reduce makespan and improves performance. The experimental results show that EACO results improved makespan.

The proposed algorithm is currently working on makespan, and it will be further extended with other functions like cost and load balancing.

\section{REFERENCES}

[1] Reddy, G. Narendrababu, and S. Phani Kumar. "MACO-MOTS: Modified Ant Colony Optimization for Multi-Objective Task Scheduling in Cloud Environment."International Journal of Intelligent Systems and Applications 11.1 (2019): 73.

[2] Sosinsky, Barrie, Cloud Computing Bible, Vol. 762, John Wiley \& Sons, 2010.

[3] Visheratin, Alexander A., Mikhail Melnik, and Denis Nasonov. "Workflow scheduling algorithms for hard-deadline constrained cloud environments." Procedia Computer Science 80 (2016): 2098- 2106.

[4] Mathew, Teena, K. Chandra Sekaran, and John Jose. "Study and analysis of various task scheduling algorithms in the cloud computing environment."Advance in Computing and Communication and Informatics (ICACCI, 2014 International Conference on. IEEE, 2014.

[5] Dorigo, Marco, and Gianni Di Caro. "Ant colony optimization: a new meta-heuristic." Proceedings of the 1999 congress on evolutionary computation-CEC99 (Cat. No. 99TH8406). Vol. 2. IEEE, 1999.

[6] Eberhart, Russell, and James Kennedy. "Particle swarm optimization." Proceeding of the IEEE international conference and neural networks. Vol. 4. 1995.

[7] Li, Kun, et al. "Cloud task scheduling based on load balancing ant colony optimization." 2011 Sixth Annual ChinaGrid Conference. IEEE, 2011.

[8] Tawfeek, Medhat A., et al. "Cloud task scheduling based on ant colony optimization." 2013 8th international conference on computer engineering \& systems (ICCES). IEEE, 2013.

[9] Moon, YoungJu, et al. "A slave ants based ant colony optimization algorithm for task scheduling in cloud computing environments." Human-centric Computing and Information Sciences 7.1 (2017): 28. 
[10] Jain, Richa, Neelam Sharma, and Pankaj Jain. "A systematic analysis of nature inspired workflow scheduling algorithm in heterogeneous cloud environment."Intelligent Communication and Computational Techniques (ICCT), 2017 International Conference on IEEE, 2017.

[11] Said, Gamal Abd El-Nasser A. "Nature Inspired Algorithms in Cloud Computing: A Survey."

[12] Saleh, Heba, et al. "IPSO Task Scheduling Algorithm for Large Scale Data in Cloud Computing Environment."IEEE Access 7 (2019): 5412-5420.

[13] Verma, Amandeep, and Sakshi Kaushal. "A hybrid multi-objective Particle Swarm Optimization for scientific workflow scheduling. "Parallel Computing 62 (2017): 1-19.

[14] Nasr, Aida A., et al. "Cost-Effective Algorithm for Workflow Scheduling in Cloud Computing Under Deadline Constraint." Arabian Journal for Science and Engineering 2018): 1-

[15] Kalra, Mala, and Sarbjeet Singh. "A review of metaheuristic scheduling techniques in cloud computing."Egyptian informatics journal 16.3 (2015): 275-295.

[16] https://www.superwits.com/library/CloudSim-simulation-framework

[17] https://opensourceforu.com/2014/03/CloudSim-framework-modelling-simulating-cloud- environment/

[18] https://www.ijcseonline.org/pub_paper/8-IJCSE-00491.pdf

[19] Tawfeek, Medhat A., et al. "An Ant Algorithm for cloud task scheduling."Proceedings of International Workshop on Cloud Computing and Information Security(CCIS). 2013.

[20] Guo, Qiang. "Task scheduling based on ant colony optimization in cloud environment." AIP Conference Proceedings. Vol. 1834. No. 1. AIP Publishing, 2017.

[21] Al-maamari, Ali, and Fatma A. Omara. "Task scheduling using PSO algorithm in cloud computing environments."International Joural of Grid and Distributed Computing 8.5 (2015): 245- 256.

[22] Verma, Amandeep, and Sakshi Kaushal. "Cost-time efficient scheduling plan for executing workflows in the cloud." Journals of grid computing 13.4 (2015): 495-506. 